\title{
Fatty-acyl Composition of the Lipids of Saccharomyces cerevisiae Grown Aerobically or Anaerobically in Media Containing Different Fatty Acids
}

\author{
By KENNETH WATSON† AND ANTHONY H. ROSE* \\ Zymology Laboratory, School of Biological Sciences, Bath University, \\ Claverton Down, Bath BA2 7 AY, Avon
}

(Received 13 September 1979)

\begin{abstract}
Saccharomyces cerevisiae grew at the same rate aerobically in a glucose/salts medium as anaerobically in a defined medium supplemented with ergosterol and either oleic, linoleic or $\alpha$-linolenic acid. However, exponential growth extended for a longer time in aerobic cultures. Irrespective of the nature of the fatty acid provided, phosphatidylcholine from anaerobically grown organisms harvested from early mid-exponential phase cultures contained a higher proportion of residues chemically identical with that provided in the medium compared with the phospholipid from organisms harvested at later stages of batch growth. The fatty-acyl composition of phosphatidylethanolamine and of a fraction containing phosphatidylinositol and phosphatidylserine showed similar changes to those found in phosphatidylcholine. Similar trends were also detected in the fatty-acyl composition of triacylglycerols from organisms grown anaerobically in the presence of different fatty acids and harvested at different stages of growth. However, at all stages of growth there was a higher percentage incorporation of the exogenously provided acid into triacylglycerols. The fatty-acyl composition of individual classes of phospholipid and of triacylglycerols from aerobically grown organisms remained remarkably constant throughout growth in batch culture. There were, however, characteristic differences in the fatty-acyl composition of different classes of lipid.
\end{abstract}

\section{INTRODUCTION}

When strains of Saccharomyces cerevisiae are grown under strictly anaerobic conditions, nutritional requirements are induced for cell components or precursors whose synthesis requires molecular oxygen. Quantitatively, the two major nutritional requirements are a sterol (Andreasen \& Stier, 1953) and an unsaturated fatty acid (Andreasen \& Stier, 1954). Minor anaerobically induced nutritional requirements, such as nicotinic acid (Suomalainen et al., 1965), are usually supplied by a low concentration of yeast extract. Requirements for both the sterol (Proudlock et al., 1968) and the unsaturated fatty acid (Light et al., 1962; Meyer et al., 1963) are fairly non-specific, findings which have been exploited in studies on mitochondrial biogenesis (Jollow et al., 1968) and, in this laboratory, in compositionfunction relationships in the plasma membrane of $S$. cerevisiae NCYC 366 (Alterthum \& Rose, 1973; Hossack \& Rose, 1976; Thomas et al., 1978; Thomas \& Rose, 1979). Apart from the report by Alterthum \& Rose (1973) that total lipids from S. cerevisiae NCYC 366 grown anaerobically in medium supplemented with oleic, linoleic or $\alpha$-linolenic acid, and harvested at a density of 0.22 to $0.24 \mathrm{mg}$ dry $\mathrm{wt} \mathrm{ml}^{-1}$, contained, respectively, $65 \cdot 1,56 \cdot 7$ and $53.9 \%$ residues of the acid supplied, nothing has been reported on the extent to which

$\dagger$ Permanent address: Department of Chemistry and Biochemistry, James Cook University of North Queensland, Townsville, Australia 4811. 
exogenous fatty acids are incorporated into different types of lipid during anaerobic growth. The present paper reports on the extent to which the major phospholipids and triacylglycerols are enriched with the exogenously provided unsaturated fatty acid (oleic, linoleic or $\alpha$-linolenic acid) at different stages of batch growth. Lipid compositions of anaerobically grown organisms are compared with those from aerobically grown cultures.

\section{METHODS}

Experimental cultures. Saccharomyces cerevisiae NCYC 366 was maintained on slopes of malt extract/ yeast extract/glucose/mycological peptone/agar medium (Wickerham, 1951). The yeast was grown anaerobically as described by Alterthum \& Rose (1973) with the medium $(\mathrm{pH} \mathrm{4.5)}$ supplemented with ergosterol $\left(5 \mathrm{mg} \mathrm{l}^{-1}\right)$ and oleic, linoleic or $\alpha$-linolenic acid $\left(30 \mathrm{mg} \mathrm{l}^{-1}\right)$. Cultures were grown aerobically as described by

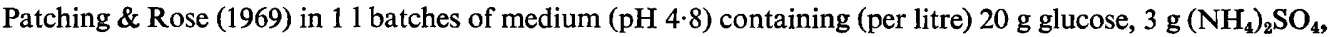
$3 \mathrm{~g} \mathrm{KH}_{2} \mathrm{PO}_{4}, 10 \mathrm{~g}$ yeast extract (Oxoid), $5 \mathrm{~g}$ mycological peptone (Oxoid), $0.025 \mathrm{~g} \mathrm{CaCl}_{2}$ and $0.025 \mathrm{~g}$ $\mathrm{MgSO}_{4} .7 \mathrm{H}_{2} \mathrm{O}$. Growth was followed by measuring turbidity at $600 \mathrm{~nm}$. Turbidity values were related to dry weight equiv. which were determined by filtering suitable volumes of culture on to preweighed filters (Millipore; $0.80 \mu \mathrm{m}$ pore size; $25 \mathrm{~mm}$ diam.), washing, and drying at $105^{\circ} \mathrm{C}$ for $24 \mathrm{~h}$.

Lipid analyses. Organisms were harvested at the culture densities indicated, washed twice with water, and lipid was extracted by a modificatinn of the Folch $e t$ al. (1957) procedure. Freshly washed organisms ( $2 \mathrm{~g}$ dry wt) were mixed with methanol $(40 \mathrm{ml})$ and the suspension was shaken in a Braun homogenizer (B. Braun, Melsungen, West Germany) for $30 \mathrm{~s}$ at speed $2\left(4000 \mathrm{rev} . \mathrm{min}^{-1}\right)$ after addition of $60 \mathrm{~g}$ glass beads (Glasperlen; B. Braun; 0.45 to $0.50 \mathrm{~mm}$ diam.). Chloroform was then added to the suspension to give the ratio $2: 1(\mathrm{v} / \mathrm{v})$ chloroform/methanol, and the suspension was stirred on a flatbed stirrer for $2 \mathrm{~h}$ at room temperature $\left(20\right.$ to $\left.22^{\circ} \mathrm{C}\right)$. The suspension was then filtered through Whatman no. 44 filter paper, and the extraction procedure was repeated on the residue. The combined extracts were washed with 0.25 vol. $0 \cdot 88 \%(\mathrm{w} / \mathrm{v}) \mathrm{KCl}$ and the mixture was separated by centrifugation or left to separate overnight at $4{ }^{\circ} \mathrm{C}$. The lower phase was removed, taken to dryness on a rotary evaporator, and the residue was immediately dissolved in chloroform/ methanol $(2: 1, \mathrm{v} / \mathrm{v})$. Samples were stored under nitrogen gas at $-20^{\circ} \mathrm{C}$.

Phospholipids were separated by thin-layer chromatography on plates of silica gel $\mathrm{H}(0.5 \mathrm{~mm}$ thick $)$ using a solvent system of chloroform/methanol/acetic acid/water (25:15:4:2, by vol.; Skipski et al., 1964). Classes of phospholipid were located by exposing plates to iodine vapour, and the positions of spots were marked with a pin. Phospholipids were identified by reference to standards run simultaneously. Using these procedures, phosphatidylinositol and phosphatidylserine were not completely separated, and fatty-acyl compositions are quoted for the combined fraction. Saccharomyces cerevisiae NCYC 366 resembles other strains of this species in containing apppreciably more phosphatidylinositol than phosphatidylserine (Ratcliffe et al., 1973). The iodine was allowed to sublime at room temperature, phospholipid-containing spots were scraped off the plate, and fatty-acid methyl esters were prepared by refluxing the silica gel with methanolic $\mathrm{HCl}$ for $2 \mathrm{~h}$. After cooling, an equal volume of water was added, and the methyl esters were extracted into hexane.

Neutral lipids were separated on plates $\left(0.4 \mathrm{~mm}\right.$ thick) of silica gel $\mathrm{HF}_{366+254}$ using a solvent system of petroleum ether (b.p. 40 to $60^{\circ} \mathrm{C}$ )/diethyl ether/acetic acid (70:30:2, by vol.). Lipids were located by examining plates under ultraviolet radiation, and triacylglycerol spots were marked with a pin. Methyl esters of fatty-acyl residues in triacylglycerols were prepared as described for phospholipids.

Fatty-acid methyl esters were analysed by gas-liquid chromatography on a column ( $2 \mathrm{~m}$ ) of $15 \%$ EGSS-Y supported on 100 to 120 mesh Gas-Chrom $P$ at $175^{\circ} \mathrm{C}$. The injection port was at $210^{\circ} \mathrm{C}$, and the carrier gas $\left(\mathrm{N}_{2}\right)$ flow rate was $40 \mathrm{ml} \mathrm{min}{ }^{-1}$. Percentage fatty-acid compositions were calculated by multiplying the retention time by the peak height on the trace.

Chemicals. Ergosterol, oleic, linoleic and $\alpha$-linolenic acids were obtained from Sigma, and phospholipids from Applied Science Laboratories, State College, Pa., U.S.A. Silica gels $\mathbf{H}$ and $\mathbf{H F}_{\mathbf{3 6 6 + 2 5 4}}$ were purchased from E. Merck. All other chemicals were reagent grade or of the highest purity available commercially.

\section{RESULTS}

\section{Growth of anaerobic and aerobic cultures}

The time-course of growth of anaerobic cultures of S. cerevisiae NCYC 366 in medium containing oleic, linoleic or $\alpha$-linolenic acid was similar (Fig. 1). Yields at the end of exponential growth were 1.5 to $1.8 \mathrm{mg}^{\text {dry }}$ wt organisms $\mathrm{ml}^{-1}$. Aerobic cultures grew at about 


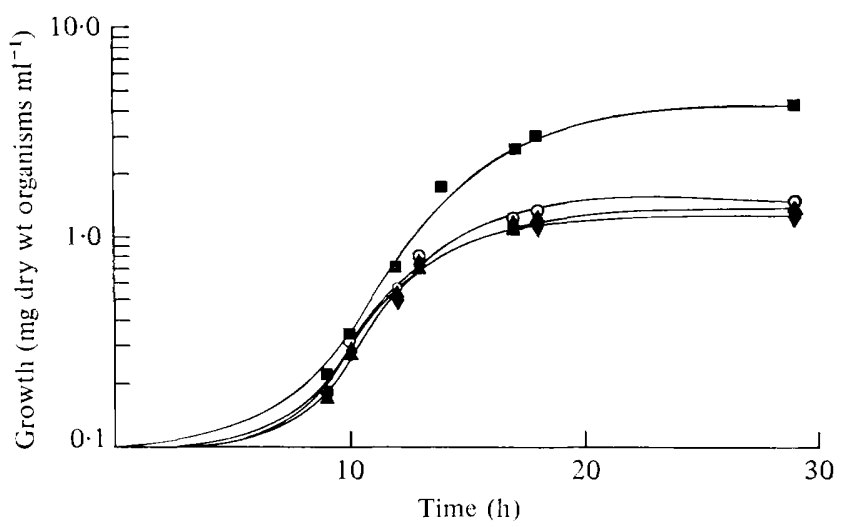

Fig. 1. Growth of Saccharomyces cerevisiae NCYC 366 aerobically ( $\square$ ), and anaerobically in medium containing oleic acid $(O)$, linoleic acid $(\boldsymbol{\Delta})$ or $\alpha$-linolenic acid $(\boldsymbol{\Delta})$. See Methods for details of media and conditions.

the same rate as anaerobic cultures, but exponential growth in aerobic cultures was more prolonged and, at the end of exponential growth, cultures contained 3.5 to $4.0 \mathrm{mg}$ dry wt organisms $\mathrm{ml}^{-1}$ (Fig. 1).

The total lipid contents of organisms grown anaerobically with any one of the fatty acids were similar to those reported by Alterthum \& Rose (1973). Organisms from earlyexponential phase aerobic cultures had a slightly higher lipid content similar to that reported by Hunter \& Rose (1972).

\section{Fatty-acyl compositions of lipids from anaerobically grown organisms}

Phosphatidylcholine. Irrespective of the nature of the exogenously provided unsaturated fatty acid, phosphatidylcholine in organisms harvested from early mid-exponential phase cultures contained a higher proportion of residues chemically identical with those provided in the medium compared with the phospholipid from organisms harvested at later stages in the growth phase (Table 1). However, the extent of enrichment with the exogenously provided acid residues was considerably greater in organisms grown in the presence of oleic acid than in the presence of $\alpha$-linolenic acid. Analyses of organisms grown in the presence of linoleic acid showed an intermediate pattern (Table 1). The decrease in the content of the exogenously provided $\mathrm{C}_{18}$ residues as growth proceeded was accompanied by an increased content of $\mathrm{C}_{16.0}$ and of shorter chain $\left(\mathrm{C}_{10: 0}, \mathrm{C}_{12: 0}\right.$ and $\left.\mathrm{C}_{14: 0}\right)$ residues.

Phosphatidylethanolamine. The fatty-acyl composition of phosphatidylethanolamine from organisms grown in the presence of different unsaturated fatty acids and harvested at various stages of growth was similar to that observed for phosphatidylcholine (Table 2). However, the changes in fatty-acyl composition of this phospholipid from organisms harvested at different stages of growth tended to be smaller than those observed with phosphatidylcholine.

Phosphatidylinositol and phosphatidylserine. The fatty-acyl composition of the fraction comprising phosphatidylinositol and phosphatidylserine showed changes in the content of the exogenously provided acid similar to those observed in phosphatidylcholine and phosphatidylethanolamine. However, the magnitude of the change in composition, as growth proceeded, was very much less marked than with the other two phospholipids, added to which the combined fraction contained a higher proportion of $\mathrm{C}_{16: 0}$ and $\mathrm{C}_{18: 0}$ residues, as reflected in the lower values for $\Delta \mathrm{mol}^{-1}$ (Table 3) compared with the other two phospholipids.

Triacylglycerols. Essentially similar trends to those reported for phospholipids were 


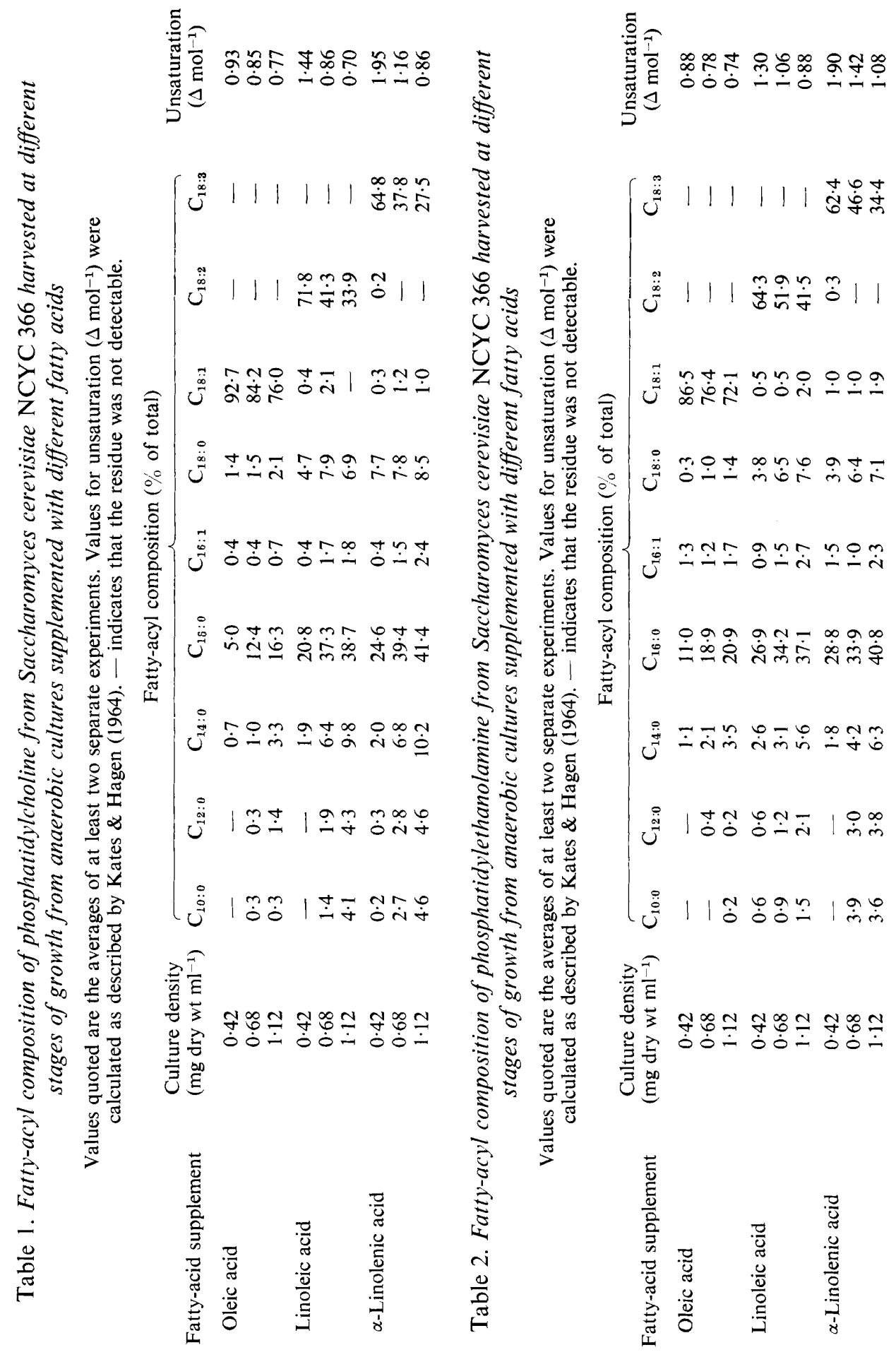


$z$
0
$\vdots$
$\vdots$
$\vdots$
0
0
0
$z$
$z$

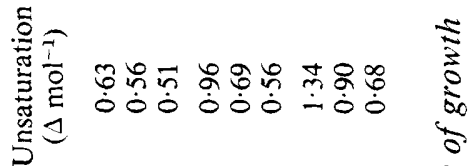

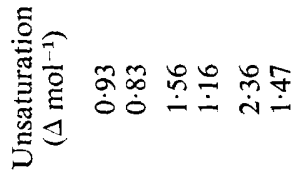

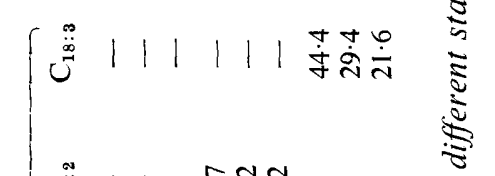

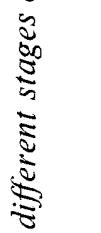

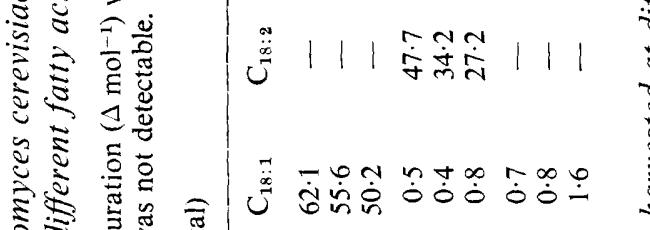

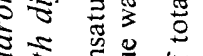

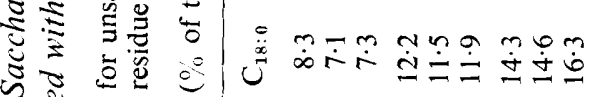

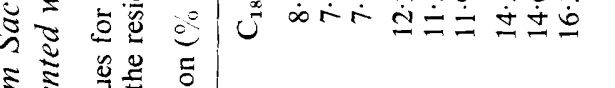

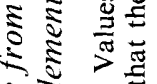

$\cong \stackrel{5}{5}$

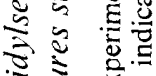

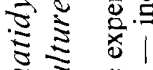

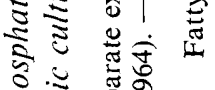

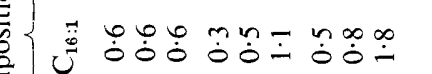

,

S

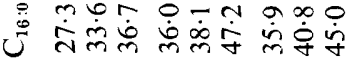

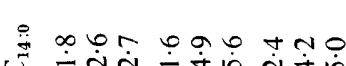

$\frac{\sqrt{0}}{3} \frac{2}{2}$

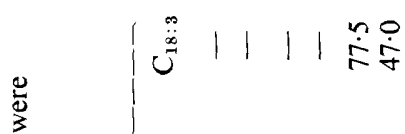

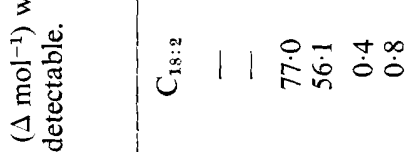

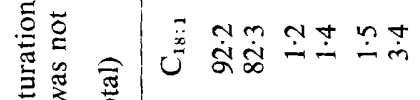

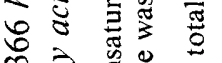

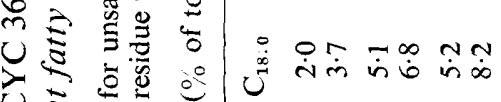

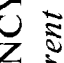

突

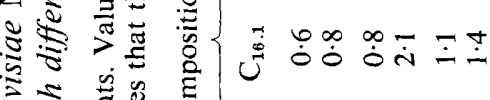

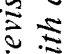

ปิ

के

ఏญ

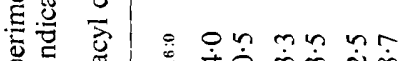

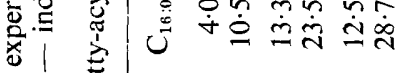

苛

ఫิ

2 离

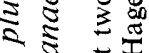

จ.

$5 \leqslant \frac{\pi}{0}$

굴

蛋

客宁

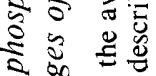

万ิ

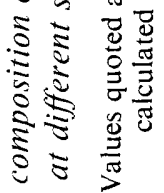

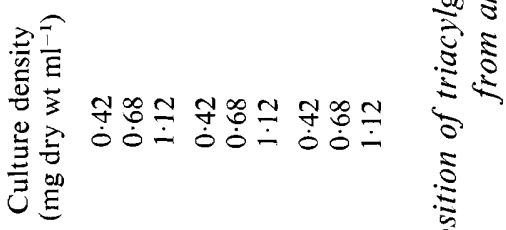

ธี

递

文芳

융

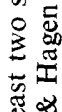

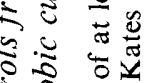

U

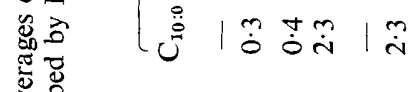

突

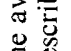

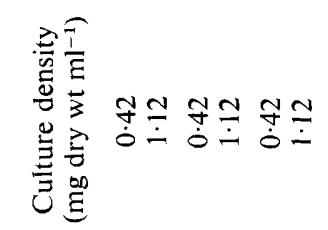

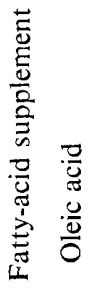

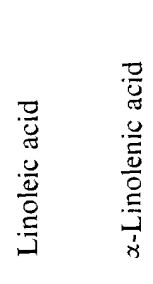

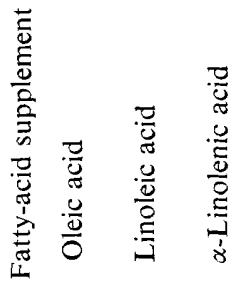




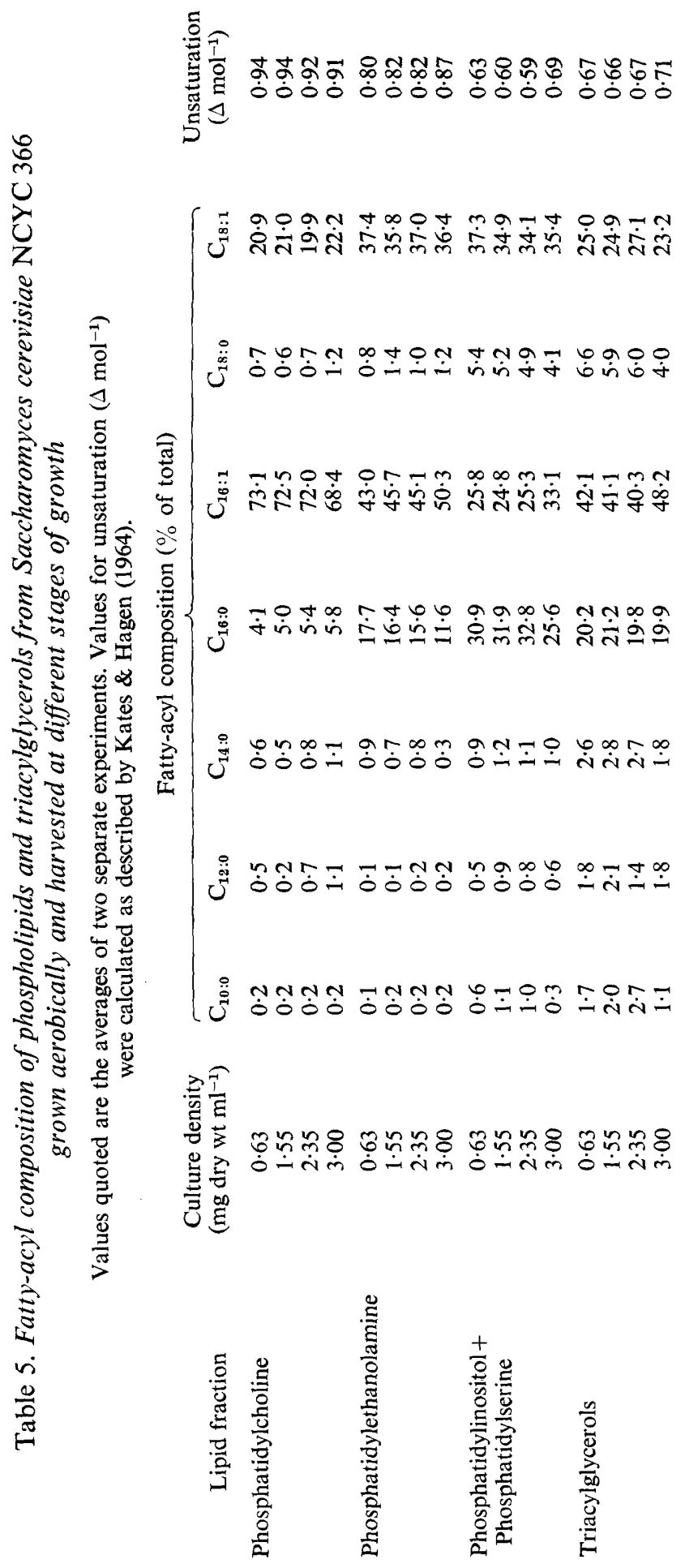


observed in the fatty-acyl composition of triacylglycerols from organisms harvested at different stages of growth. However, there was a higher percentage incorporation of the exogenously provided acid at all stages of growth (Table 4).

\section{Fatty-acyl composition of lipids from aerobically grown organisms}

Compared with lipids from anaerobically grown organisms, the composition of phospholipids and triacylglycerols from aerobically grown organisms remained remarkably constant throughout growth in batch culture (Table 5). There was, however, a marked variation in the fatty-acyl composition of the different lipids. Phosphatidylcholine remained highly unsatured, with palmitoleyl and oleyl residues predominant. These two residues also predominated in phosphatidylethanolamine, but in lower proportions, as indicated by the overall lower $\Delta \mathrm{mol}^{-1}$ value for this phospholipid. The fraction comprising phosphatidylinositol and phosphatidylserine contained a much higher proportion of saturated residues, particularly $\mathrm{C}_{16: 0}$, compared with the other two phospholipids. Triacylglycerols had a similar overall $\Delta \mathrm{mol}^{-1}$ value to the mixture of phospholipids in the combined fraction, but differed in their contents of $\mathrm{C}_{16: 1}$ and $\mathrm{C}_{18: 1}$ residues.

\section{DISCUSSION}

Yeast phospholipids are located almost exclusively in membranes. Changes in the degree of unsaturation of phospholipids will therefore alter the fluidity of the membrane, that is the extent to which phospholipid fatty-acyl chains are mobile in the bilayer. Changes in unsaturation could also affect the activity of membrane-bound enzymes and proteins concerned with transport processes, since individual proteins may have requirements for individual phospholipid molecules with specific unsaturated fatty-acyl residues, as already reported for certain bacterial membrane-bound enzymes (Finnerty, 1979). Lipids of $S$. cerevisiae NCYC 366 grown anaerobically in the presence of oleic acid had a fluidity (as judged by the $\Delta \mathrm{mol}^{-1}$ value) which was reasonably constant throughout growth in batch culture. Unsaturation values for phosphatidylcholine and phosphatidylethanolamine in these organisms were similar to those reported for these phospholipids from aerobically grown organisms and are typical for phospholipids from yeasts grown in the range 25 to $30^{\circ} \mathrm{C}$ (Kates \& Baxter, 1962; Hunter \& Rose, 1972; Erwin, 1973). On the other hand, comparable values for unsaturation in phospholipids from organisms grown in the presence of linoleic or $\alpha$-linolenic acid were only observed with lipids from late-exponential phase organisms. Exceptionally high unsaturation values were detected in phospholipids from organisms grown in the presence of multiply unsaturated acids and harvested earlier during exponential growth. Such high values for lipid unsaturation have generally only been recorded for psychrophilic yeasts (Kates \& Baxter, 1962; Arthur \& Watson, 1976; Watson et al., 1978) when grown at suboptimum temperatures, when the high degree of lipid unsaturation is likely to be essential to maintain membranes in a functional state.

It might be inferred from these findings that, in organisms grown in the presence of linoleic or $\alpha$-linolenic acid, membrane fluidity during the early- and mid-exponential growth phases might be so high as to render membranes unstable. The comparable rates of growth and final yields of organisms observed with anaerobic cultures containing each of the three different unsaturated fatty acids indicate that membrane instability is not sufficient to affect basic growth parameters. There is, however, evidence for instability in plasma membranes in S. cerevisiae NCYC 366 containing multiply unsaturated fatty-acyl residues. Sphaeroplasts prepared from cells enriched in either linoleic or $\alpha$-linolenic acid residues leaked protein faster than sphaeroplasts with membranes enriched in oleyl residues (Diana M. Belk \& A. H. Rose, unpublished observations). Furthermore, $\alpha$-mannosyltransferase activities of plasma-membrane preparations were more variable when the membranes were enriched in linoleyl rather than oleyl residues, which might be explained by a greater loss of 
components (protein and/or phospholipid) from the more fluid membranes (L. Julia Douglas, unpublished observations). Finally, M. H. J. Keenan \& A. H. Rose (unpublished observations) detected a greater rate of decay of certain transport systems in $S$. cerevisiae NCYC 366 with membranes enriched in linoleyl rather than oleyl residues, which again might be explained by loss of proteins from the more fluid membranes.

There are several possible ways in which $S$. cerevisiae growing anaerobically in the presence of multiply unsaturated fatty acids might attempt to minimize fluidity in cellular membranes. One strategem would be to incorporate, preferentially, exogenously provided multiply unsaturated fatty acids into neutral lipids (e.g. triacylglycerols) which are not membrane components. Significantly, triacylglycerols from anaerobically grown organisms were more unsaturated than the phospholipids from these organisms. A second possibility is that decreased fluidity could arise as a result of an increased synthesis of shorter chain fatty-acyl residues, which was observed in organisms grown in the presence of multiply unsaturated fatty acids. Yet another mechanism whereby membrane fluidity could be balanced is through synthesis of phosphatidylinositol and phosphatidylserine, with a higher proportion of saturated fatty-acyl residues, strategically located in the membrane thereby maintaining a degree of rigidity in that domain.

Finally, our data on differences in the fatty-acyl composition of phospholipids from anaerobically and aerobically grown organisms could indicate operation of different biosynthetic pathways in organisms grown under these two conditions. Two pathways for synthesis of phosphatidylcholine are known to operate in S. cerevisiae (Steiner \& Lester, 1972; Waechter \& Lester, 1973), namely the methylation pathway in which phosphatidylcholine arises by methylation of phosphatidylethanolamine, and the cytidine nucleotide pathway in which free choline or ethanolamine is incorporated into phospholipids. The dissimilarity of the fatty-acyl composition of phospholipids from aerobically grown organisms could be indicative of a biosynthetic pathway in which specific phosphatidic acid species are used for synthesis of particular phospholipid classes. On the other hand, under anaerobic conditions with lipid supplements, it might appear that phosphatidylcholine and phosphatidylethanolamine are synthesized from a common pool of phosphatidic acid.

This work was carried out with financial assistance from the Science Research Council (U.K.) under research grant no. GR/A/41564, for which we express our gratitude.

\section{REFERENCES}

Alterthum, F. \& Rose, A. H. (1973). Osmotic lysis of sphaeroplasts from Saccharomyces cerevisiae grown anaerobically in media containing different unsaturated fatty acids. Journal of General Microbiology 77, 371-382.

Andreasen, A. A. \& Stier, T. J. B. (1953). Anaerobic nutrition of Saccharomyces cerevisiae. I. Ergosterol requirement for growth in a defined medium. Journal of Cellular and Comparative Physiology 41, 23-36.

Andreasen, A. A. \& Stier, T. J. B. (1954). Anaerobic nutrition of Saccharomyces cerevisiae. II. Unsaturated fatty acid requirement for growth in a defined medium. Journal of Cellular and Comparative Physiology 43, 271-281.

Arthur, H. \& Watson, K. (1976). Thermal adaptation in yeast: growth temperatures, membrane lipid and cytochrome composition of psychrophilic, mesophilic and thermophilic yeasts. Journal of Bacteriology 128, 56-68.

ERwiN, J. A. (1973). Comparative biochemistry of fatty acids in eukaryotic microorganisms. In Lipids and Biomembranes of Eukaryotic Microorganisms, pp. 41-143. Edited by J. A. Erwin. New York: Academic Press.

FinNerTy, W. R. (1979). Physiology and biochemistry of bacterial phospholipid metabolism. Advances in Microbial Physiology 18, 177-233.

Folch, J., Lees, M. \& Sloane Stanley, G. H. (1957). A simple method for the isolation and purification of total lipids from animal tissues. Journal of Biological Chemistry 226, 497-509.

Hossack, J. A. \& Rose, A. H. (1976). Fragility of plasma membranes in Saccharomyces cerevisiae enriched with different sterols. Journal of Bacterio$\log y$ 127, 67-75.

Hunter, K. \& Rose, A. H. (1972). Lipid composition of Saccharomyces cerevisiae as influenced by growth temperature. Biochimica et biophysica acta 260, 639-653.

Jollow, D., Kellerman, G. M. \& Linnane, A. W. (1968). The biogenesis of mitochondria. III. The 
lipid composition of aerobically and anaerobically grown Saccharomyces cerevisiae as related to the membrane systems of the cells. Journal of Cell Biology 37, 221-230.

Kates, M. \& Baxter, R. M. (1962). Lipid composition of mesophilic and psychrophilic yeasts (Candida sp.) as influenced by environmental temperature. Canadian Journal of Biochemistry and Physiology 40, 1213-1227.

Kates, M. \& HaGen, P. O. (1964). Influence of temperature on fatty acid composition of psychrophilic and mesophilic Serratia spp. Canadian Journal of Biochemistry 42, 481-488.

Light, R. J., LenNarz, W. J. \& Bloch, K. (1962). The metabolism of hydroxystearic acids in yeast. Journal of Biological Chemistry 237, 1793-1800.

Meyer, F., Light, R. J. \& Bloch, K. (1963). Fatty acid metabolism in anaerobic yeast. Journal of Biological Chemistry 238, 2654-2659.

Patching, J. W. \& Rose, A. H. (1969). The effects and control of temperature. Methods in Microbiology 2, 23-38.

Proudlock, J. W., Wheeldon, L. W., Jollow, D. J. \& LinNane, A. W. (1968). Role of sterols in Saccharomyces cerevisiae. Biochimica et biophysica acta 152, 434-437.

Ratcliffe, S. J., Hossack, J. A., Wheeler, G. E. \& Rose, A. H. (1973). Modification of the phospholipid composition of Saccharomyces cerevisiae induced by exogenous ethanolamine. Journal of General Microbiology 76, 445-449.

Skipski, V. P., Peterson, R. F. \& Barclay, M. (1964). Quantitative analysis of phospholipids by thin-layer chromatography. Biochemical Journal 90, 374-384.
SteINeR, M. R. \& LeSTER, R. L. (1972). In vitro studies of phospholipid biosynthesis in Saccharomyces cerevisiae. Biochimica et biophysica acta 260, 222-243.

Suomalainen, H., Nurminen, T., BihervaARa, K. \& OURA, E. (1965). Effect of aeration on the synthesis of nicotinic acid and nicotinamide adenine nucleotide by baker's yeast. Journal of the Institute of Brewing 71, 227-231.

Thomas, D. S. \& Rose, A. H. (1979). Inhibitory effect of ethanol on growth and solute accumulation by Saccharomyces cerevisiae as affected by plasma-membrane lipid composition. Archives of Microbiology 122, 49-55.

Thomas, D. S., Hossack, J. A. \& Rose, A. H. (1978). Plasma-membrane lipid composition and ethanol tolerance in Saccharomyces cerevisiae. Archives of Microbiology 117, 239-245.

WAechter, C. J. \& Lester, R. L. (1973). Differential regulation of the $N$-methyl transferase responsible for phosphatidylcholine synthesis in Saccharomyces cerevisiae. Archives of Biochemistry and Biophysics 158, 401-410.

Watson, K., Morton, H., Arthur, H. \& Streamer, K. (1978). Membrane lipid composition: a determinant of anaerobic growth and petite formation in psychrophilic and psychrophobic yeasts. Biochemical Society Transactions 6, 380-381.

Wickerham, L. J. (1951). Taxonomy of yeasts. I. Techniques of classification. United States Department of Agriculture Technical Bulletin, no. 1029. Washington, D.C.: U.S. Department of Agriculture. 